\title{
Oxime-Based Receptors for Mono- und Disaccharides
}

\author{
Monika Mazik* and Arno C. Buthe
}

Page

1. ${ }^{1} \mathrm{H}$ and ${ }^{13} \mathrm{C}$ NMR Spectra of compounds $\mathbf{1 a}, \mathbf{1 b}, \mathbf{2 a}$ and $\mathbf{2 b}$.

2. Representative mole ratio plot.

3. Crystal data for compound 1a.

4. Crystal data for compound $\mathbf{1 b}$.

4. Description of a titration experiment with dodecyl $\beta$-D-maltoside (3).

6. Description of a titration experiment with octyl $\beta$-D-glucopyranoside (5) 
1. ${ }^{1} \mathrm{H}$ and ${ }^{13} \mathrm{C}$ NMR Spectra of compounds $\mathbf{1 a}, \mathbf{1 b}, \mathbf{2 a}$ and $\mathbf{2 b}$.
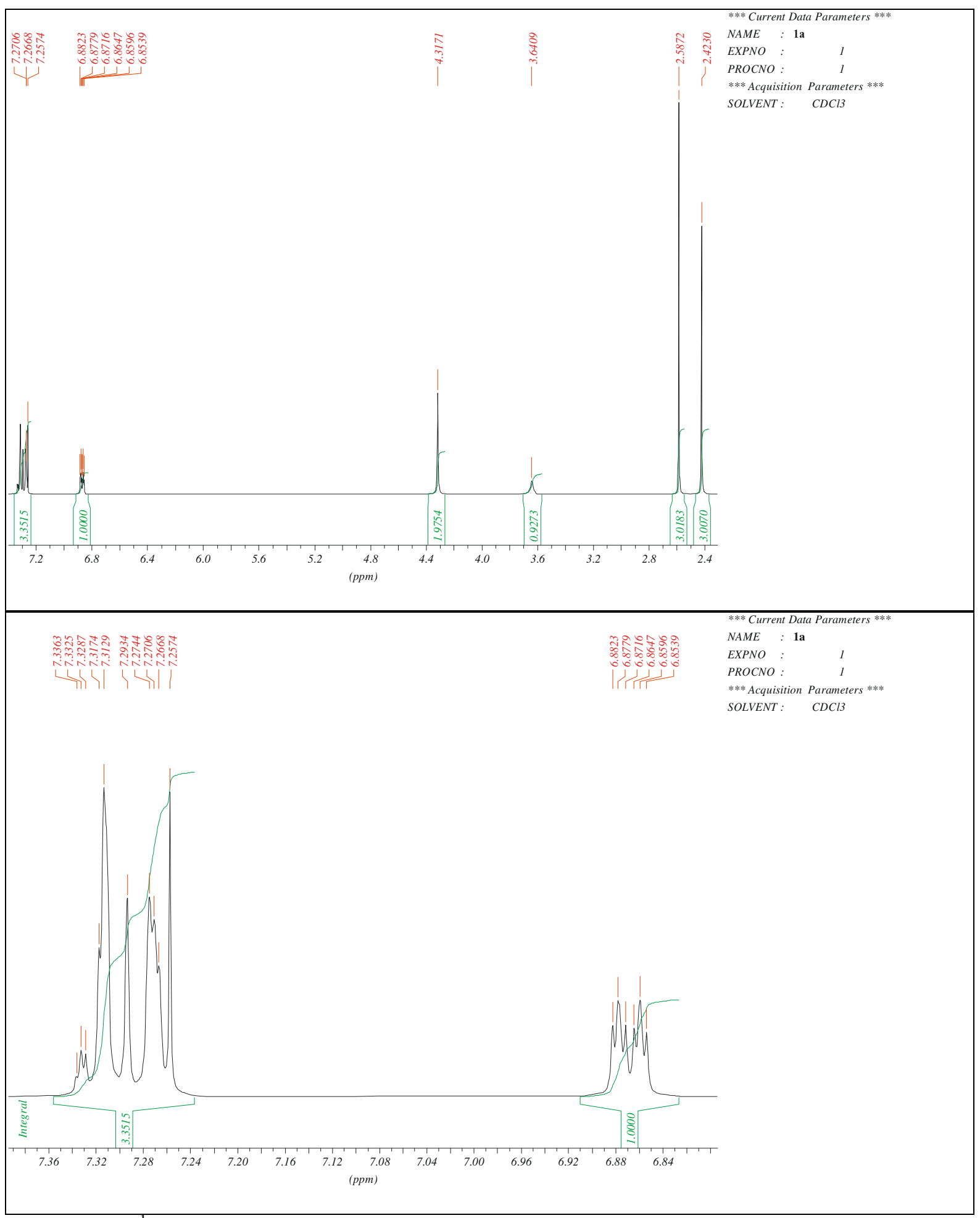

FIGURE S1. ${ }^{1} \mathrm{H}$ NMR Spectrum of $\mathbf{1 a}\left(\mathrm{CDCl}_{3}, 400 \mathrm{MHz}\right)$. 


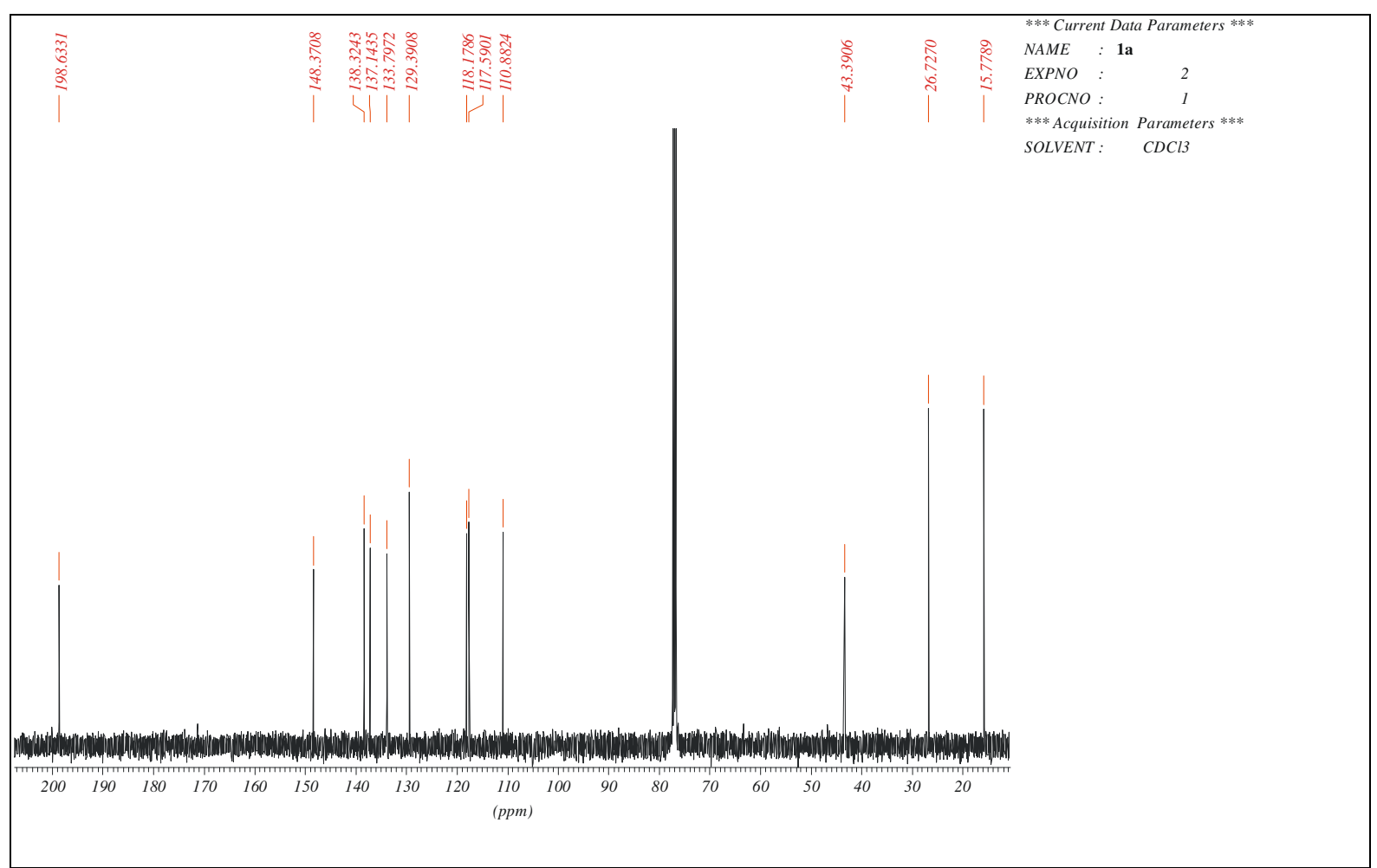

FIGURE S2. ${ }^{13} \mathrm{C}$ NMR Spectrum of $1 \mathrm{a}\left(\mathrm{CDCl}_{3}\right)$.

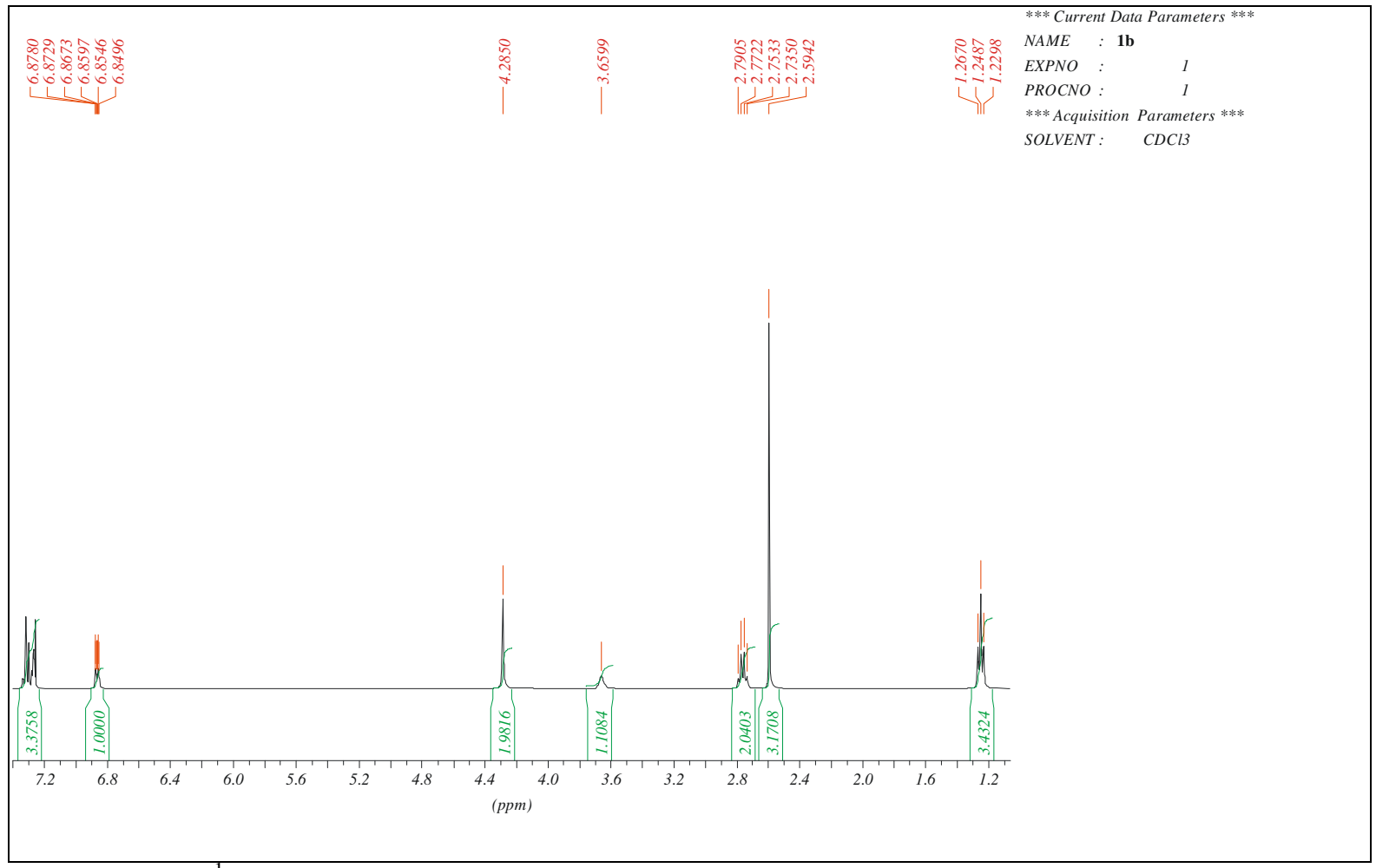

FIGURE S3a. ${ }^{1} \mathrm{H}$ NMR Spectrum of $\mathbf{1 b}\left(\mathrm{CDCl}_{3}, 400 \mathrm{MHz}\right)$. 


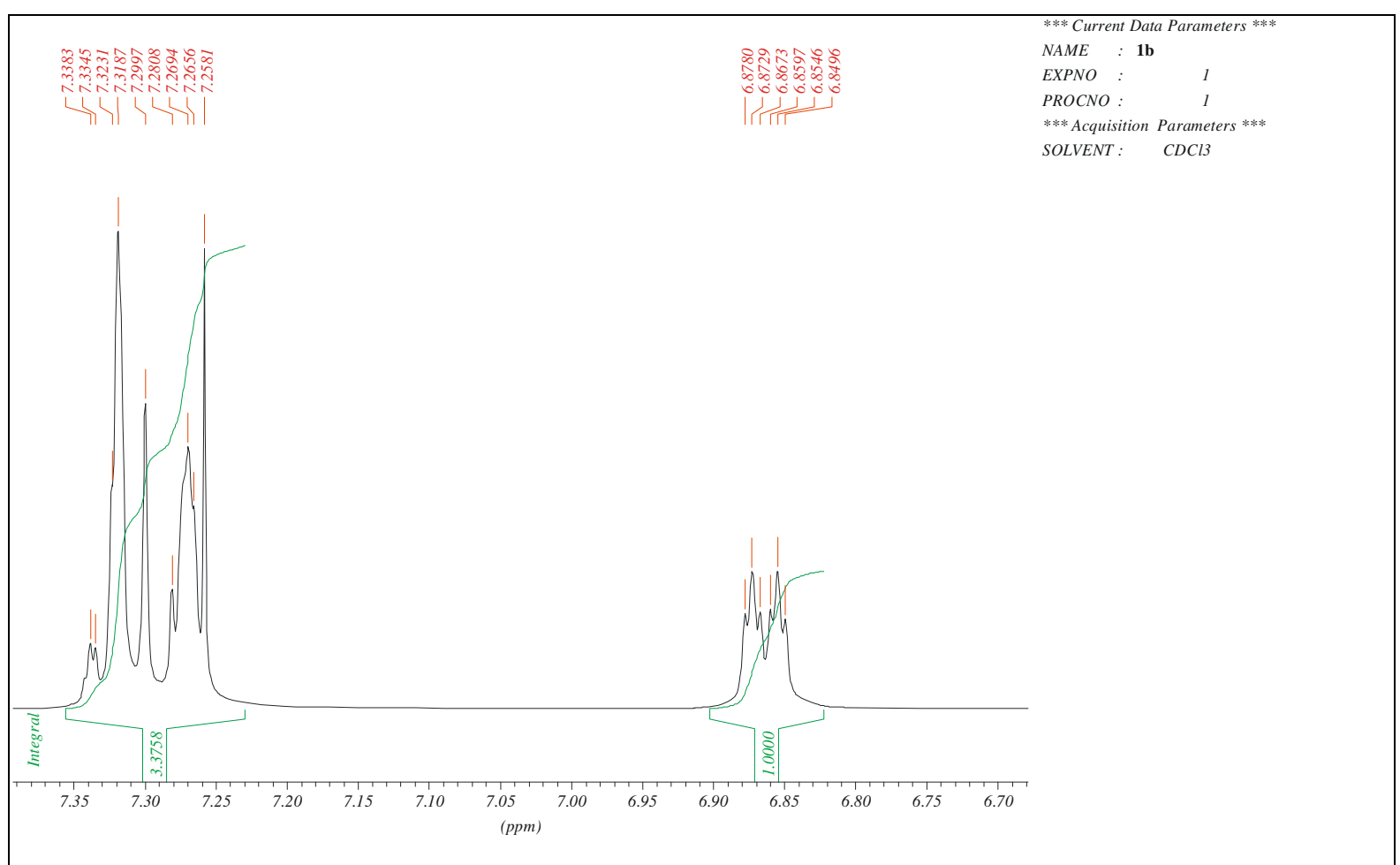

FIGURE S3b. Partial ${ }^{1} \mathrm{H}$ NMR Spectrum of $\mathbf{1 b}\left(\mathrm{CDCl}_{3}, 400 \mathrm{MHz}\right)$.

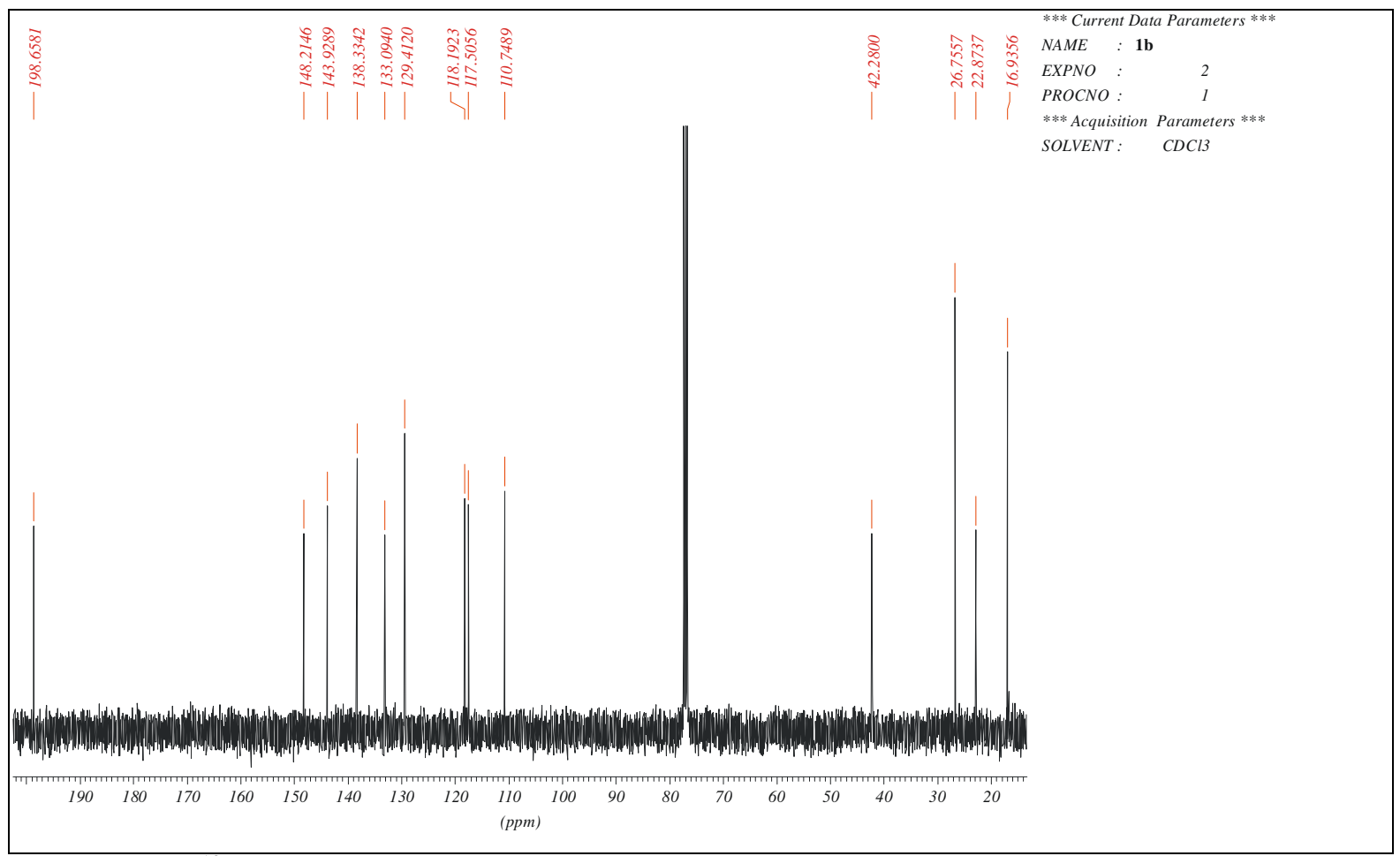

FIGURE S4. ${ }^{13} \mathrm{C}$ NMR Spectrum of $\mathbf{1 b}\left(\mathrm{CDCl}_{3}\right)$. 


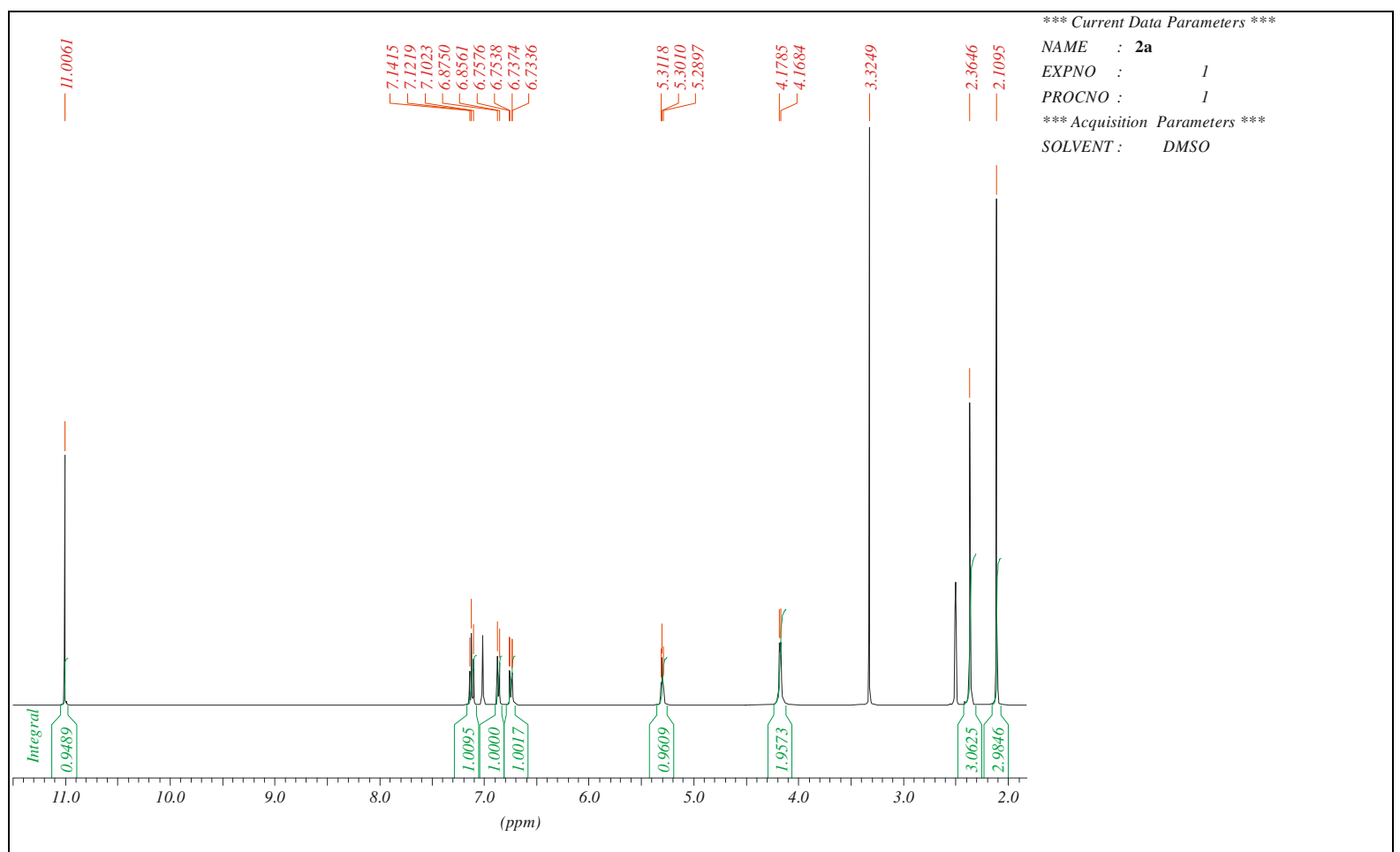

FIGURE S5a. ${ }^{1} \mathrm{H}$ NMR Spectrum of 2a $\left(\right.$ DMSO- $\left._{6}, 400 \mathrm{MHz}\right)$.

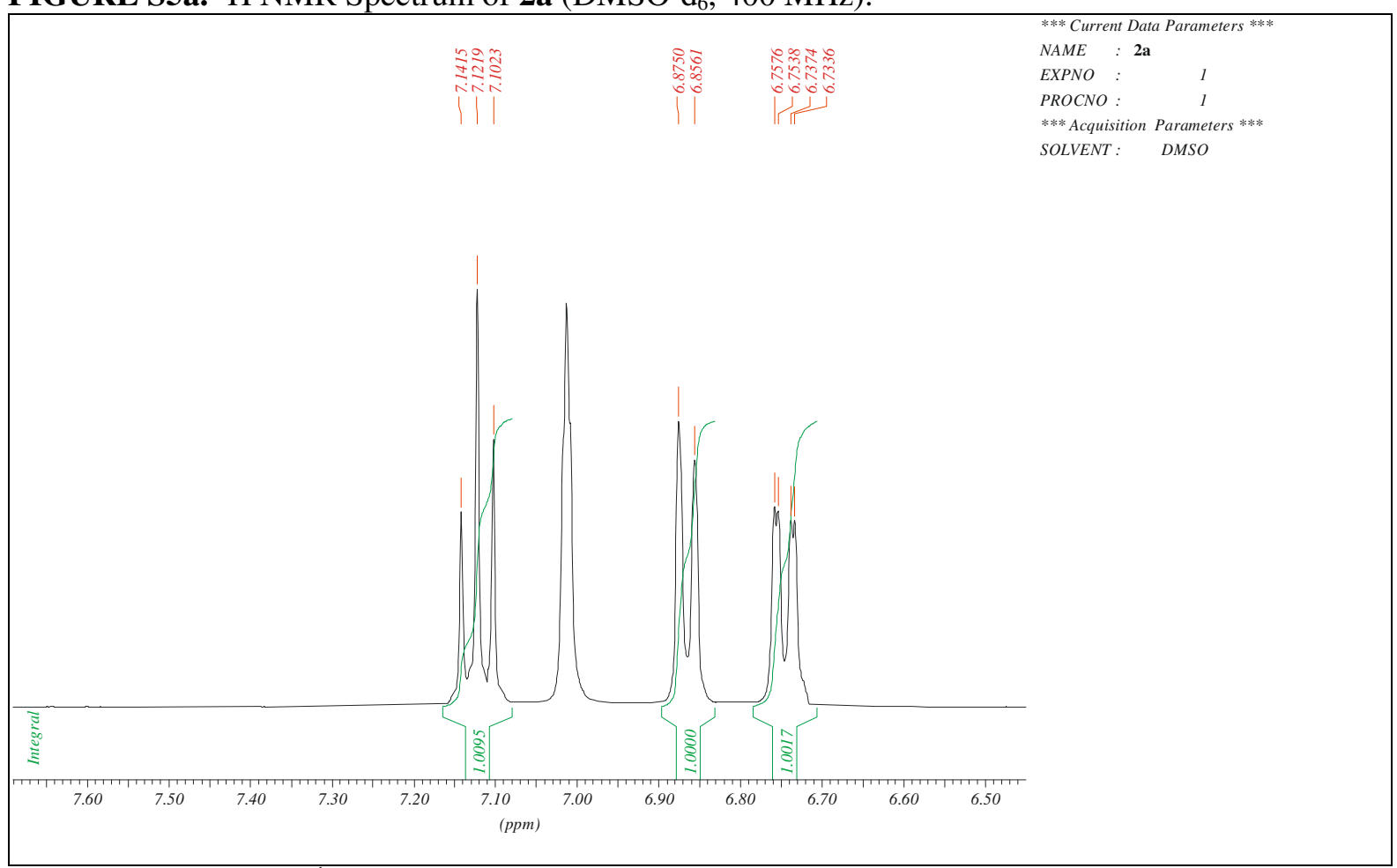

FIGURE S5b. Partial ${ }^{\mathrm{I}} \mathrm{H}$ NMR Spectrum of 2a $\left(\mathrm{DMSO}^{-} \mathrm{d}_{6}, 400 \mathrm{MHz}\right)$. 


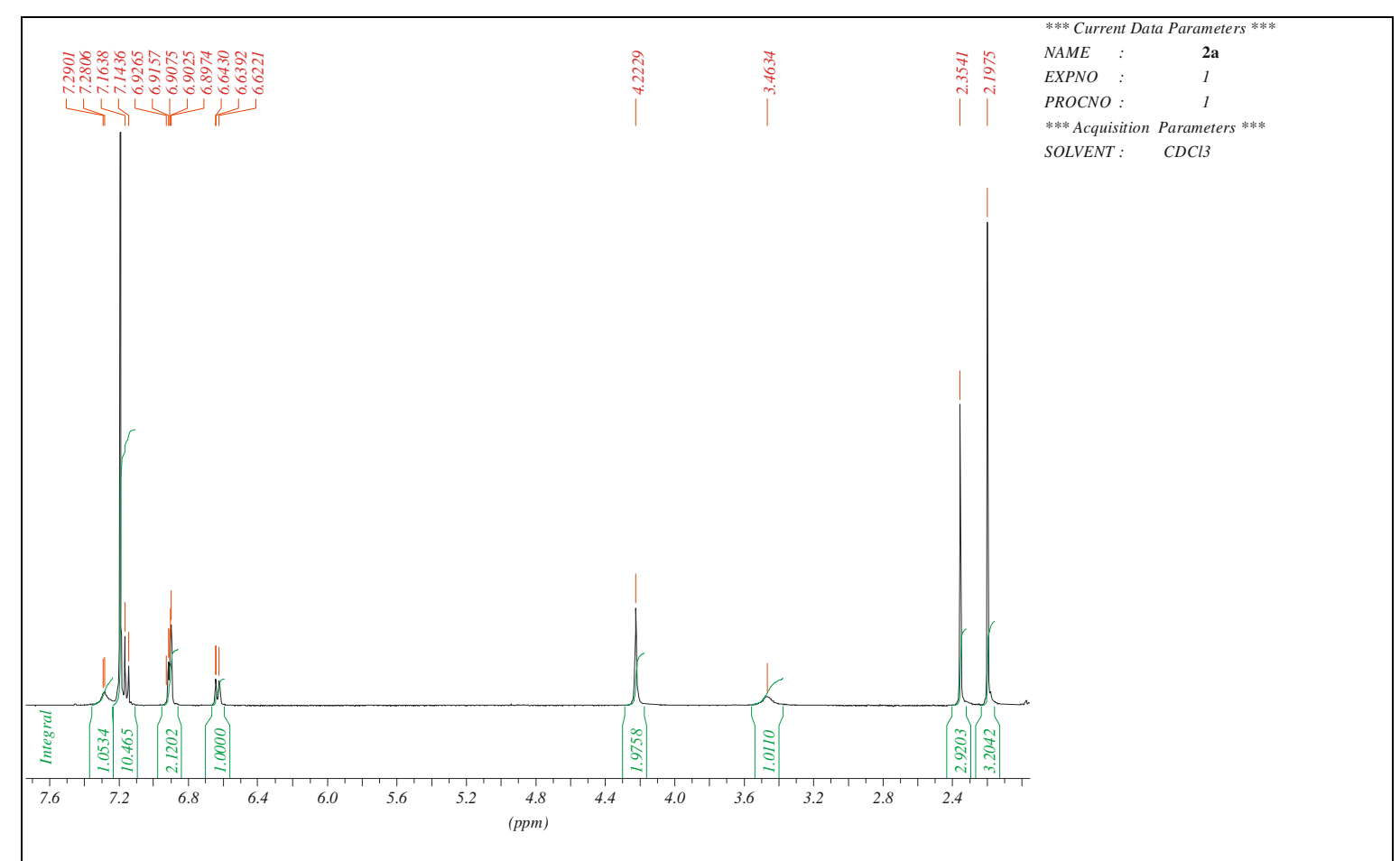

FIGURE S6a. ${ }^{1} \mathrm{H}$ NMR Spectrum of 2a $\left(\mathrm{CDCl}_{3}, 400 \mathrm{MHz}\right.$; [2a] = $\left.0.9 \mathrm{mM}\right)$.

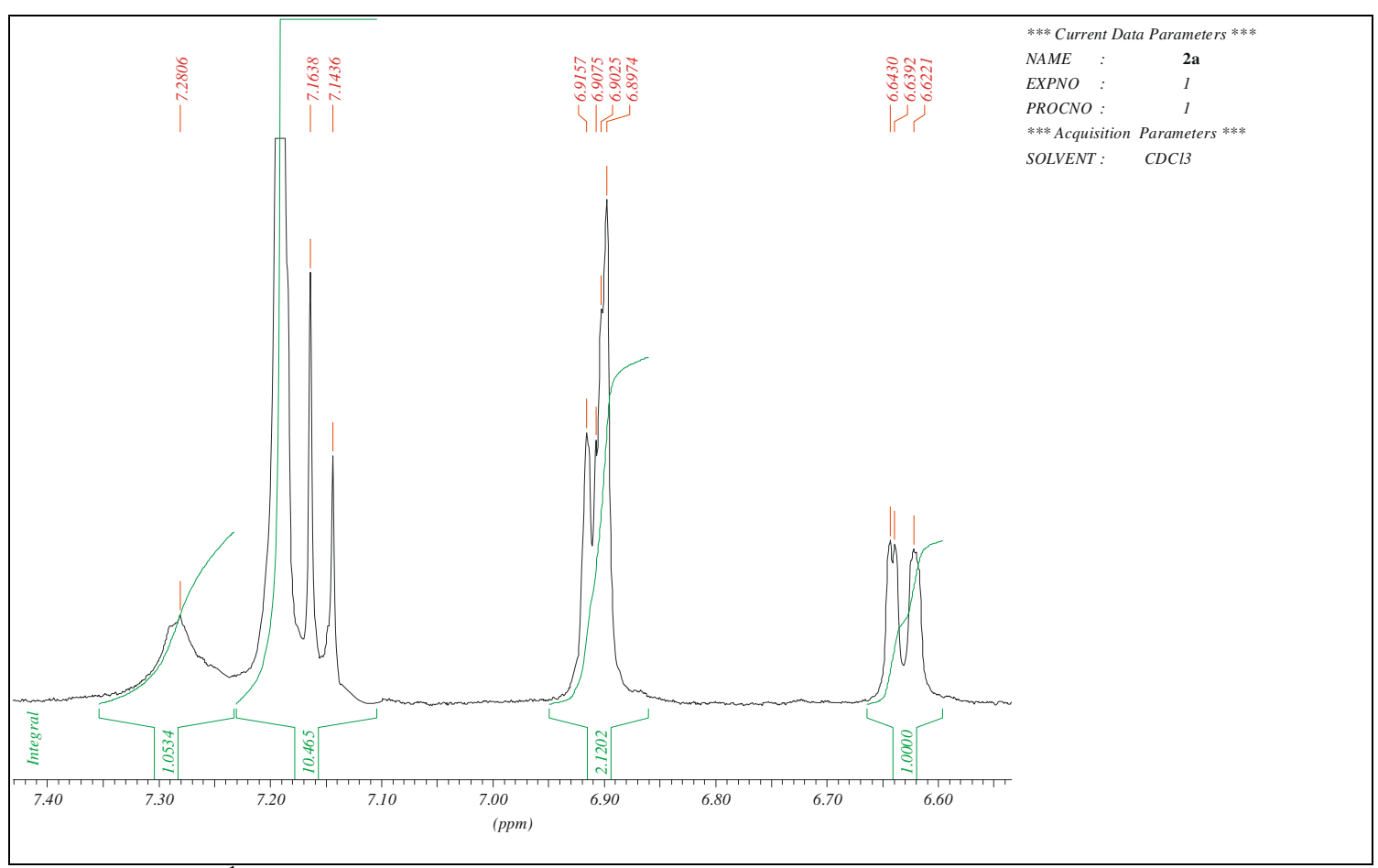

FIGURE S6b. ${ }^{1} \mathrm{H}$ NMR Spectrum of $\mathbf{2 a}\left(\mathrm{CDCl}_{3}, 400 \mathrm{MHz}\right.$; [2a] = $\left.0.9 \mathrm{mM}\right)$. 


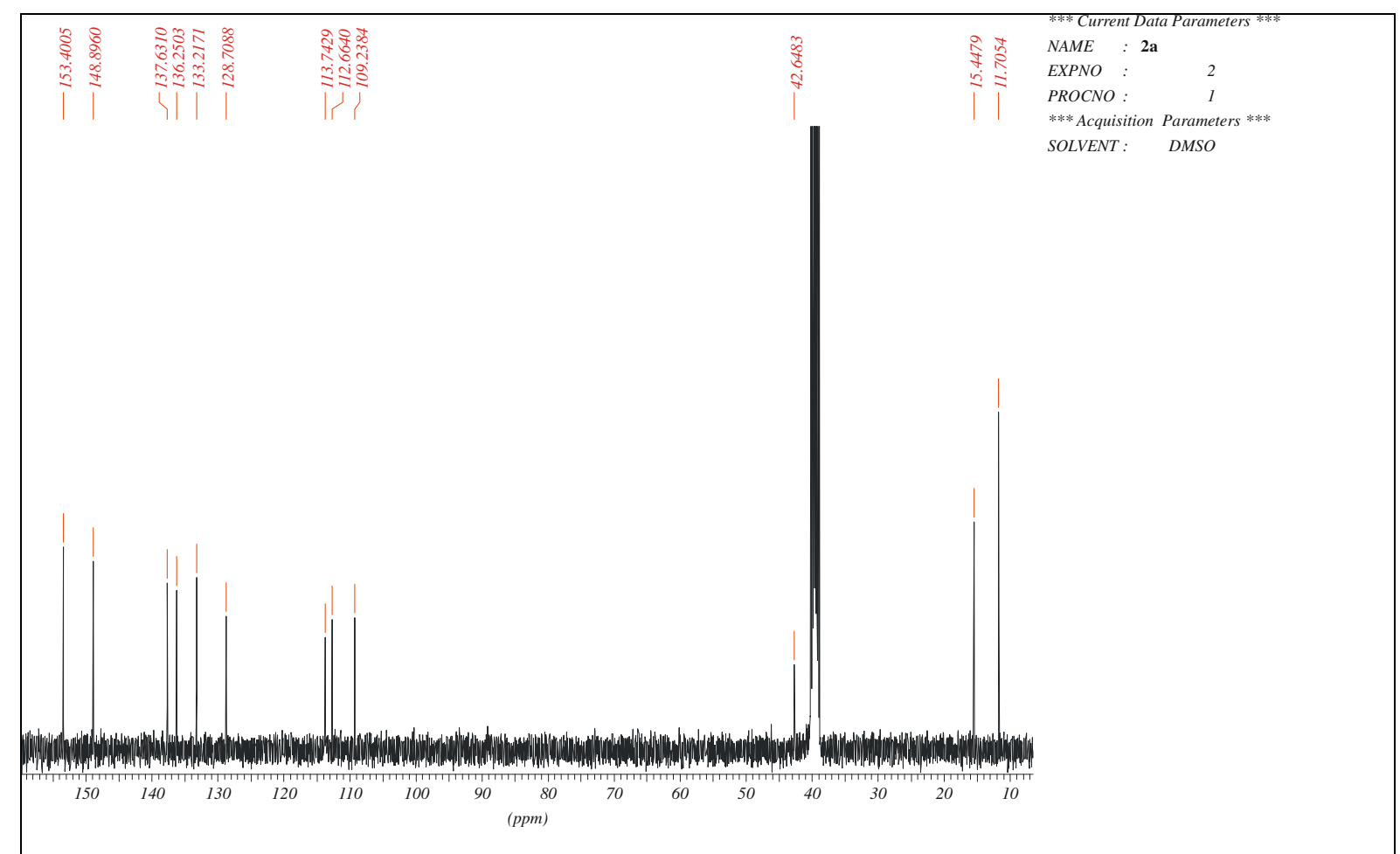

FIGURE S7. ${ }^{13} \mathrm{C}$ NMR Spectrum of $\mathbf{2 a}\left(\mathrm{DMSO}-\mathrm{d}_{6}\right)$.

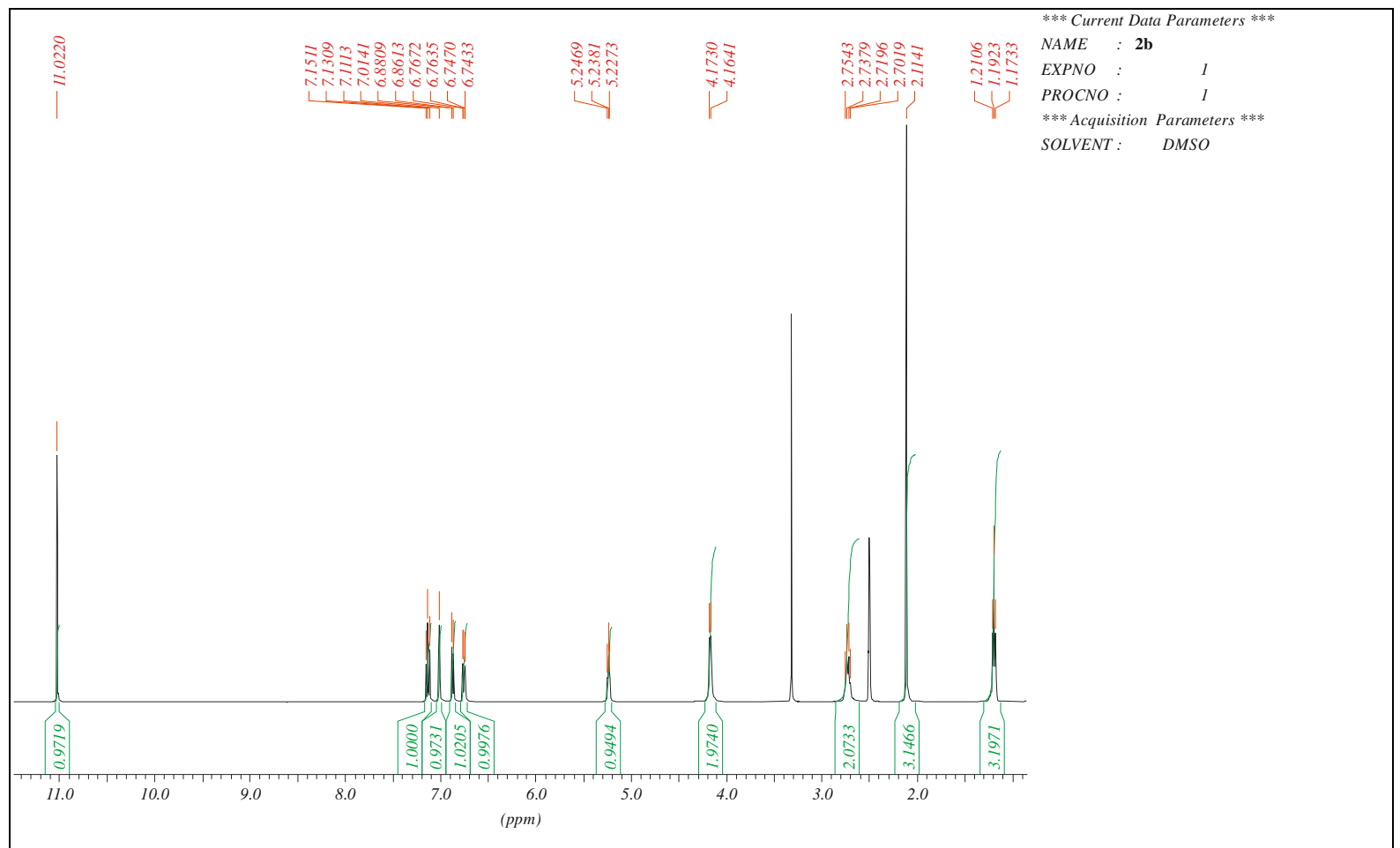

FIGURE S8a. ${ }^{1} \mathrm{H}$ NMR Spectrum of $2 \mathbf{b}\left(\mathrm{DMSO}^{\mathrm{d}} \mathrm{d}_{6}, 400 \mathrm{MHz}\right)$. 


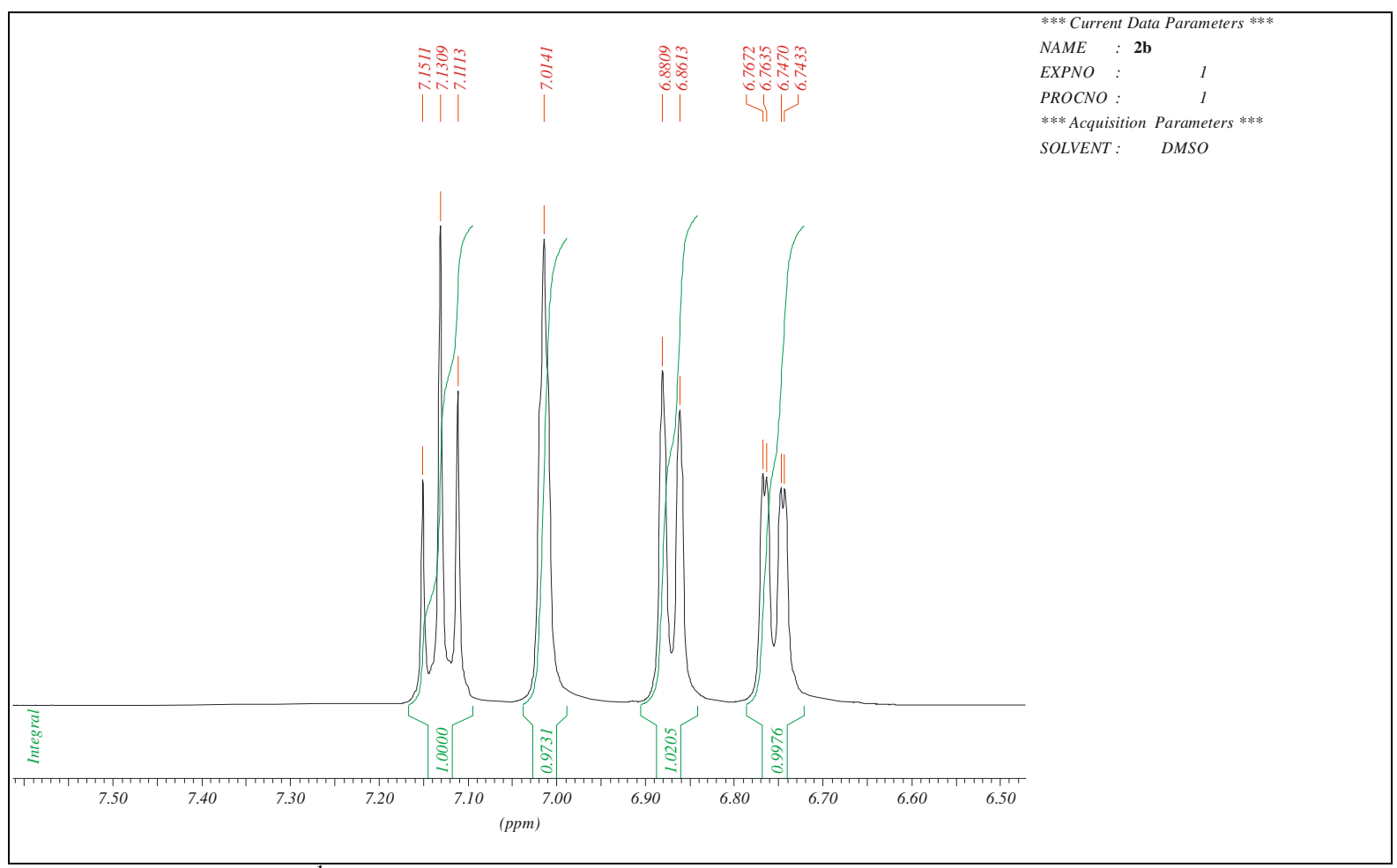

FIGURE S8b. Partial ${ }^{1} \mathrm{H}$ NMR Spectrum of $\mathbf{2 b}\left(\mathrm{DMSO}_{-} \mathrm{d}_{6}, 400 \mathrm{MHz}\right)$.

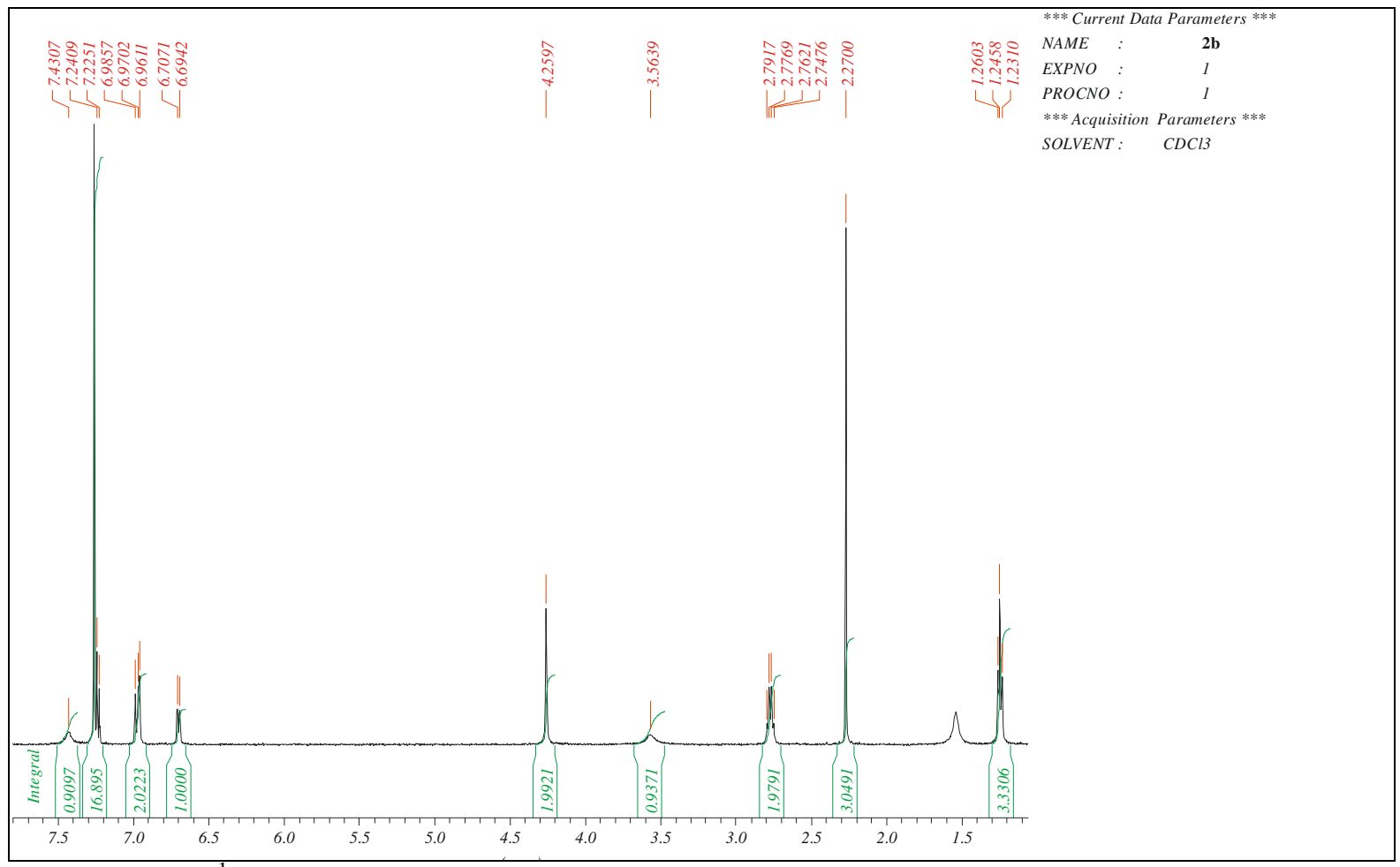

FIGURE S9a. ${ }^{1} \mathrm{H}$ NMR Spectrum of $\mathbf{2 b}\left(\mathrm{CDCl}_{3}, 400 \mathrm{MHz} ;[\mathbf{2 b}]=0.9 \mathrm{mM}\right)$. 


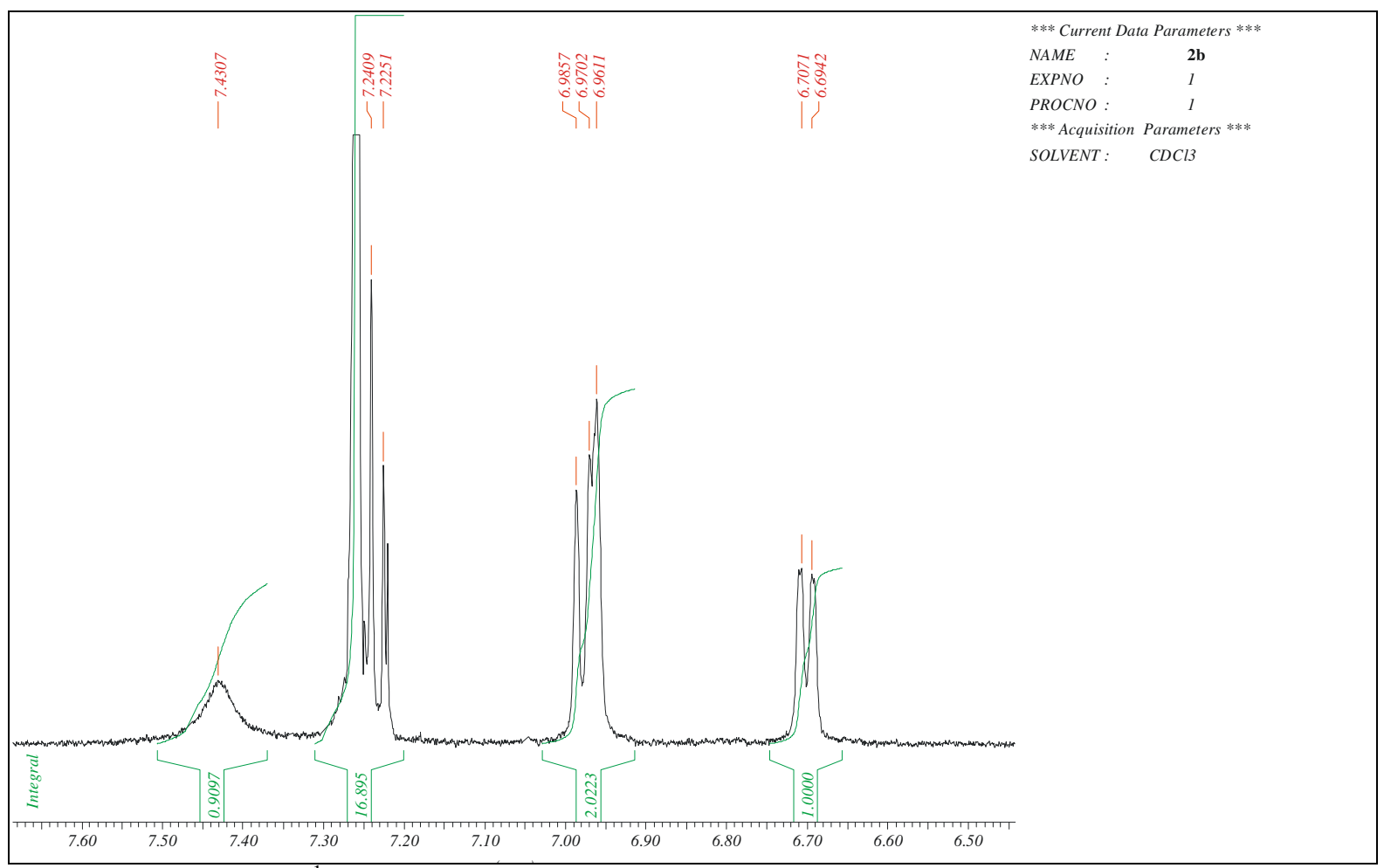

FIGURE S9b. Partial ${ }^{1} \mathrm{H}$ NMR Spectrum of $\mathbf{2 b}\left(\mathrm{CDCl}_{3}, 400 \mathrm{MHz} ;[\mathbf{2 b}]=0.9 \mathrm{mM}\right)$.

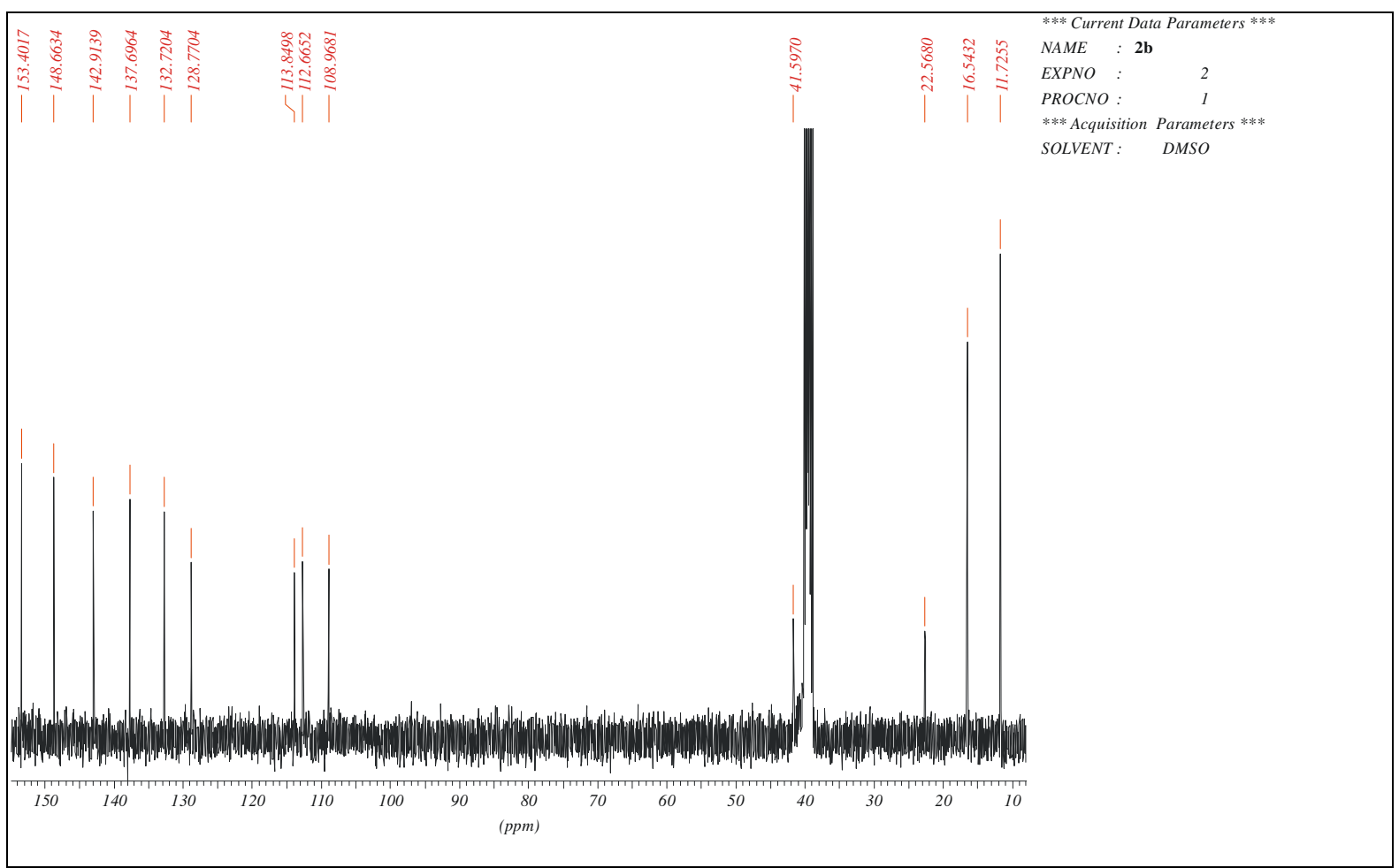

FIGURE S10. ${ }^{13} \mathrm{C}$ NMR Spectrum of $2 \mathbf{b}\left(\mathrm{DMSO}_{-} \mathrm{d}_{6}\right)$. 
2. Representative mole ratio plot.

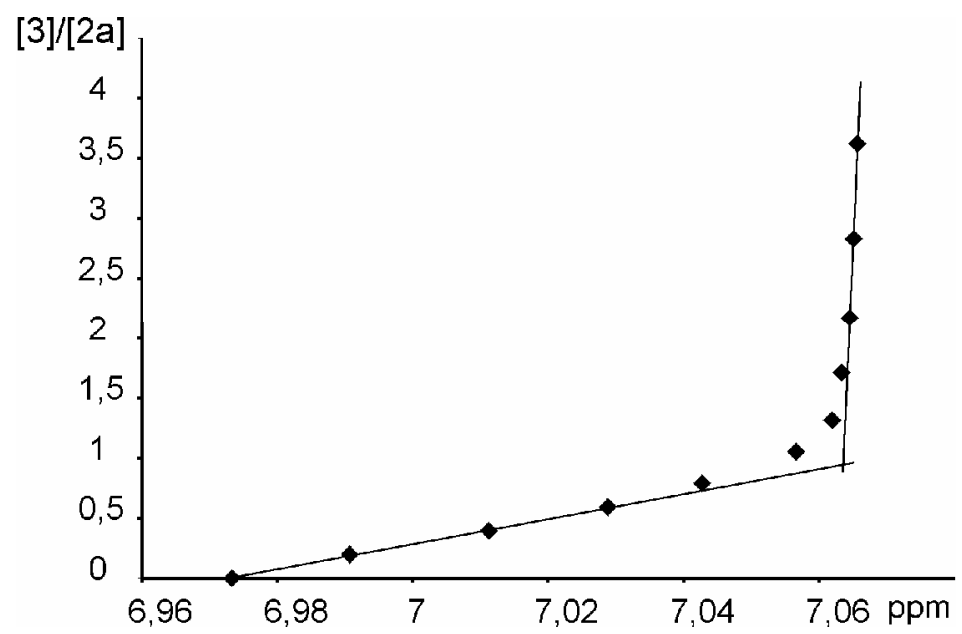

FIGURE S11. Mole ratio plot. Titration of 2a with maltoside 3 (analysis of the shifts of the $\mathrm{CH}$ protons of $\mathbf{2 a})$.

3 Crystal data for compound 1a.

Table 1. Crystal data and structure refinement.

Identification code

Empirical formula

Formula weight

Temperature

Wavelength

Crystal system

Space group

Unit cell dimensions

Volume

Z

Density (calculated)

Absorption coefficient

$\mathrm{F}(000)$

Crystal size

Theta range for data collection

Index ranges

Reflections collected

Independent reflections

Completeness to theta $=30.00^{\circ}$

Absorption correction

Max. and min. transmission

Refinement method

Data / restraints / parameters

Goodness-of-fit on F2 tube (compound 1a)

$\mathrm{C}_{38} \mathrm{H}_{41} \mathrm{Cl}_{6} \mathrm{~N}_{3} \mathrm{O}_{3}$

800.44

133(2) K

$0.71073 \AA$

Monoclinic

$\mathrm{P} 2 / \mathrm{n}$

$a=16.8041(11) \AA \quad \alpha=90^{\circ}$

$\mathrm{b}=13.6025(8) \AA \quad \beta=90.073(4)^{\circ}$

$c=16.8563(11) \AA \quad \gamma=90^{\circ}$

3853.0(4) $\AA^{3}$

4

$1.380 \mathrm{Mg} / \mathrm{m}^{3}$

$0.487 \mathrm{~mm}^{-1}$

1664

$0.50 \times 0.33 \times 0.24 \mathrm{~mm}^{3}$

1.71 to $30.52^{\circ}$

$-23<=\mathrm{h}<=23,-19<=\mathrm{k}<=19,-24<=\mathrm{k}<=24$

81810

$11744[\mathrm{R}$ (int) $=0.0285]$

$100.0 \%$

Semi-empirical from equivalents

0.8922 and 0.7967

Full-matrix least-squares on $\mathrm{F}^{2}$

11744 / 3 / 469

1.050 
Final $R$ indices [l>2sigma(I)]

$R$ indices (all data)

Largest diff. peak and hole
$\mathrm{R} 1=0.0500, \mathrm{wR} 2=0.1183$

$\mathrm{R} 1=0.0784, \mathrm{wR} 2=0.1449$

1.061 and -1.065 e. $\AA^{-3}$

4 Crystal data for compound $\mathbf{1 b}$.

Table 1. Crystal data and structure refinement.

Identification code

Empirical formula

Formula weight

Temperature

Wavelength

Crystal system

Space group

Unit cell dimensions

Volume

Z

Density (calculated)

Absorption coefficient

$\mathrm{F}(000)$

Crystal size

Theta range for data collection

Index ranges

Reflections collected

Independent reflections

Completeness to theta $=28.00^{\circ}$

Absorption correction

Refinement method

Data / restraints / parameters

Goodness-of-fit on $\mathrm{F}^{2}$

Final $R$ indices [ $>2$ sigma(I)]

$R$ indices (all data)

Largest diff. peak and hole butolu (compound 1b)

$$
\mathrm{C}_{39} \mathrm{H}_{45} \mathrm{~N}_{3} \mathrm{O}_{3}
$$

603.78

133(2) K

$0.71073 \AA$

Rhombohedral

$\mathrm{R}(-3)$

$\begin{array}{ll}a=17.5611(11) \AA & \alpha=90^{\circ} \\ b=17.5611(11) \AA & \beta=90^{\circ} \\ c=19.5070(18) \AA & \gamma=120^{\circ}\end{array}$

5209.8(7) $\AA^{3}$

6

$1.155 \mathrm{Mg} / \mathrm{m}^{3}$

$0.073 \mathrm{~mm}^{-1}$

1944

$0.3 \times 0.2 \times 0.2 \mathrm{~mm}^{3}$

1.70 to $28.28^{\circ}$

$-23<=\mathrm{h}<=23,-23<=\mathrm{k}<=23,-25<=\mathrm{k}<=25$

17452

$2865[R$ (int) $=0.0493]$

$100.0 \%$

None

Full-matrix least-squares on $\mathrm{F}^{2}$

2865 / 0 / 142

1.061

$\mathrm{R} 1=0.0618, \mathrm{wR} 2=0.1490$

$\mathrm{R} 1=0.0839, \mathrm{wR} 2=0.1587$

0.501 and -0.298 e. $\AA^{-3}$ 
5. Description of a titration experiment with dodecyl $\beta$-D-maltoside (2 $\beta$ ).

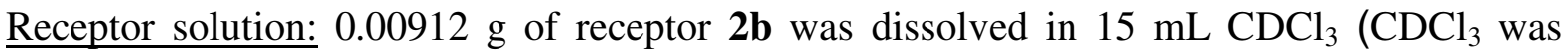
deacidified with $\mathrm{Al}_{2} \mathrm{O}_{3}$ )

Sugar solution: $0.01415 \mathrm{~g}$ of maltoside 3 was dissolved in $6.5 \mathrm{~mL}$ of the receptor solution

\begin{tabular}{|l|c|c|c|}
\hline & Receptor solution $[\mu \mathrm{L}]$ & $\begin{array}{c}\text { Solution of maltoside 3 in } \\
\text { the receptor solution }[\mu \mathrm{L}]\end{array}$ & $\begin{array}{c}\text { [maltoside]:[receptor] } \\
\text { ratio }\end{array}$ \\
\hline 1 & 900 & 0 & 0.15 \\
\hline 2 & 870 & 30 & 0.30 \\
\hline 3 & 840 & 60 & 0.45 \\
\hline 4 & 810 & 90 & 0.60 \\
\hline 5 & 780 & 120 & 0.80 \\
\hline 6 & 740 & 160 & 1.01 \\
\hline 7 & 700 & 200 & 1.31 \\
\hline 8 & 640 & 260 & 1.66 \\
\hline 9 & 570 & 330 & 2.17 \\
\hline 10 & 470 & 430 & 2.78 \\
\hline 11 & 350 & 550 & 3.13 \\
\hline 12 & 280 & 620 & 3.63 \\
\hline 13 & 180 & 720 & 4.04 \\
\hline 14 & 100 & 800 & 4.29 \\
\hline 15 & 50 & 850 & 4.54 \\
\hline 16 & -- & 900 & \\
\hline
\end{tabular}

6. Description of a titration experiment with octyl $\beta$-D-glucopyranoside (5).

Receptor solution: $0.01306 \mathrm{~g}$ of receptor $\mathbf{2 b}$ was dissolved in $10 \mathrm{~mL} \mathrm{CDCl}_{3}\left(\mathrm{CDCl}_{3}\right.$ was deacidified with $\mathrm{Al}_{2} \mathrm{O}_{3}$ )

Sugar solution: $0.03402 \mathrm{~g}$ of 5 was dissolved in $5 \mathrm{~mL} \mathrm{CDCl}_{3}$

\begin{tabular}{|l|c|c|c|}
\hline & Receptor solution $[\mu \mathrm{L}]$ & Sugar solution $[\mu \mathrm{L}]$ & $\mathrm{CDCl}_{3}[\mu \mathrm{L}]$ \\
\hline 1 & 500 & 0 & 500 \\
\hline 2 & 500 & 20 & 480 \\
\hline 3 & 500 & 40 & 460 \\
\hline 4 & 500 & 70 & 430 \\
\hline 5 & 500 & 80 & 420 \\
\hline 6 & 500 & 120 & 380 \\
\hline 7 & 500 & 160 & 340 \\
\hline 8 & 500 & 190 & 310 \\
\hline 9 & 500 & 220 & 280 \\
\hline 10 & 500 & 260 & 240 \\
\hline 11 & 500 & 310 & 190 \\
\hline 12 & 500 & 360 & 140 \\
\hline 13 & 500 & 390 & 110 \\
\hline 14 & 500 & 430 & 70 \\
\hline 15 & 500 & 470 & 30 \\
\hline
\end{tabular}

